\title{
Pâturer : regards agronomiques et pratiques d'éleveurs
}

La conduite du pâturage comme révélateur des stratégies d'éleveurs dans le Livradois-Forez

Grazing management, an indicator of livestock farmers' strategies in the

Livradois-Forez

Isabelle Boisdon, Claire Balay, Jean-Yves Pailleux, Nathalie Hostiou, Hélène Rapey et Sylvie Cournut

\section{OpenEdition \\ Journals}

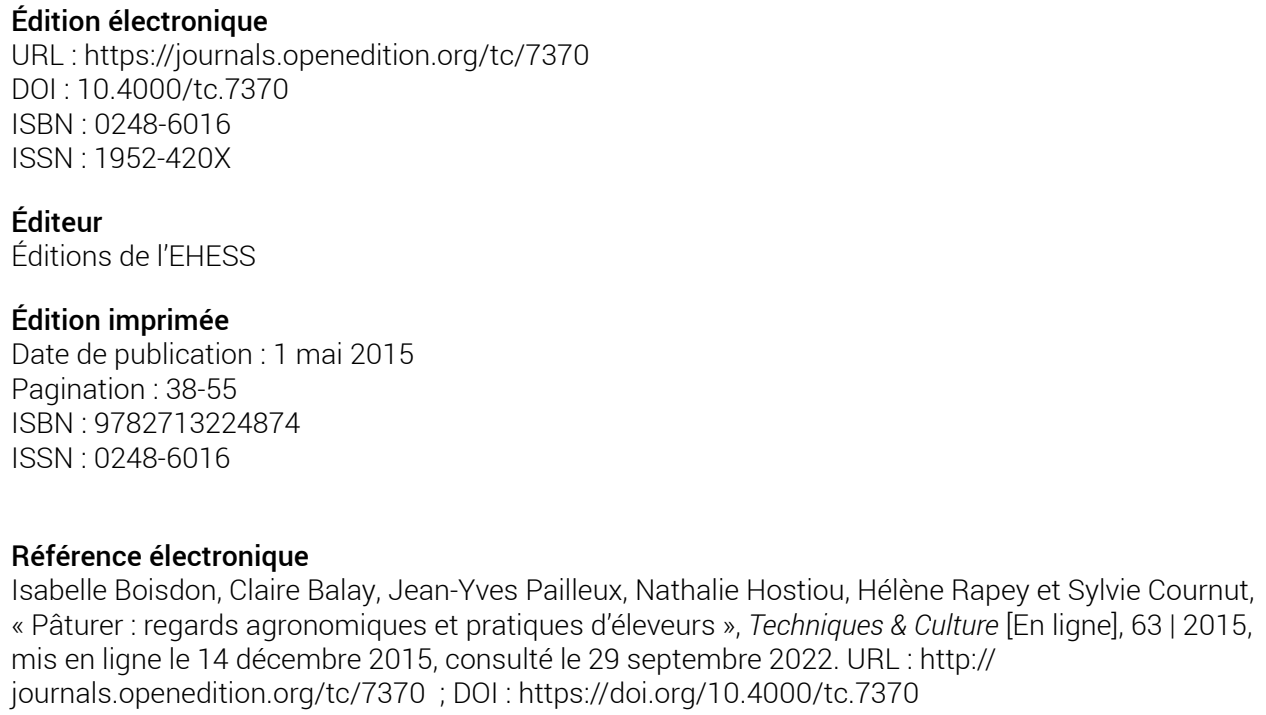




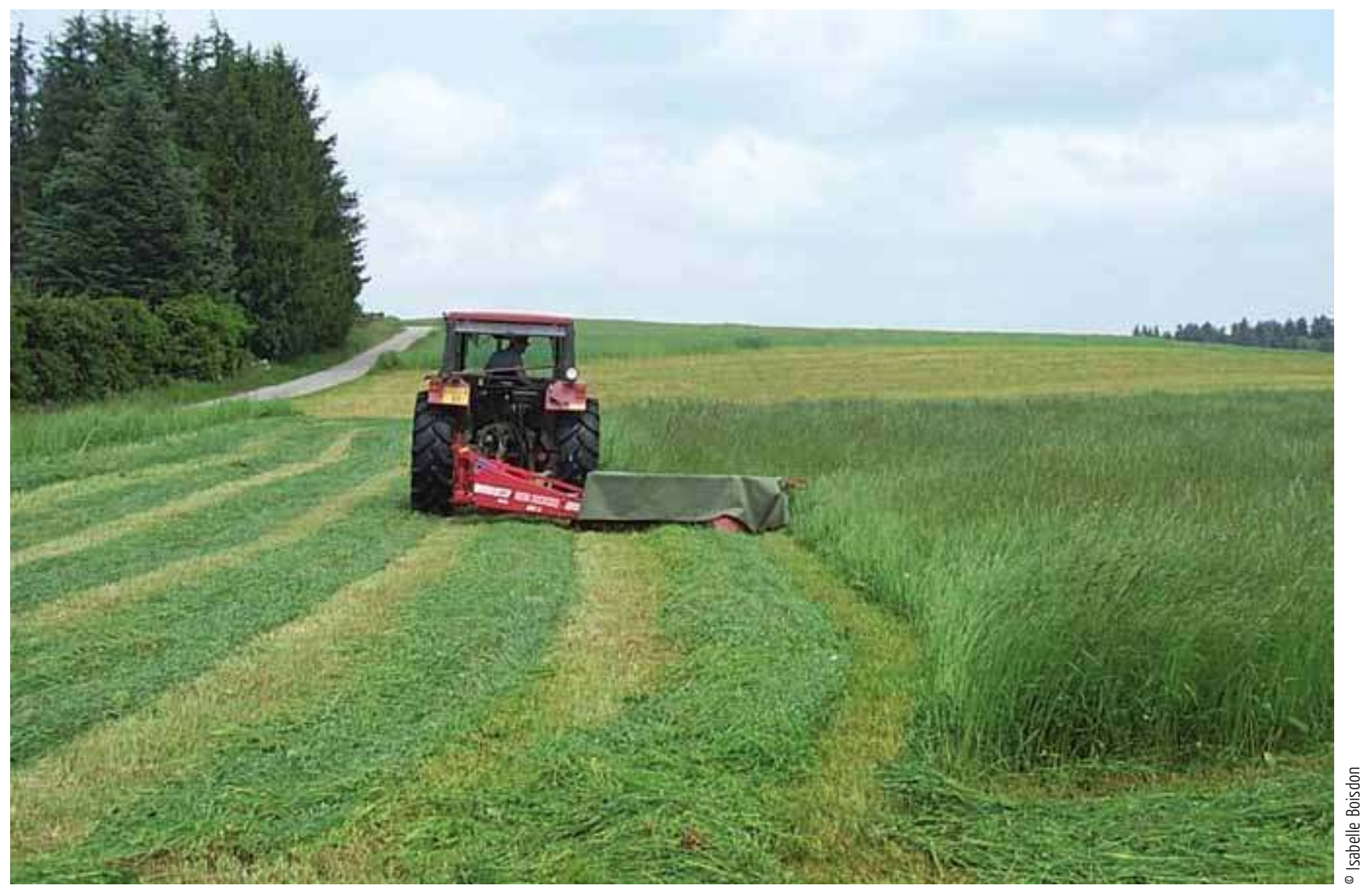




\section{PÂTURER: REGARDS AGRONOMIQUES ET PRATIQUES D'ÉLEVEURS}

\section{La conduite du pâturage comme révélateur des stratégies d'éleveurs dans le Livradois-Forez}

La maîtrise des rations alimentaires des animaux a été, avec la génétique et la médecine vétérinaire, à la base des accroissements de productivité réalisés dans les élevages français depuis les années soixante. Lorsque les fourrages pâturés sont une composante significative des rations, cette maîtrise implique de gérer des équilibres complexes en « temps réel » entre les disponibilités en fourrage et les besoins des animaux. Ainsi, comprendre les modes de gestion de l'alimentation au pâturage et les conforter nécessite un regard appuyé sur les conceptions et les pratiques des agriculteurs (Darré \& al., 1993, ; Darré $\&$ al., 2004 ; Mathieu, 2004) et s'accompagne d'un ensemble de travaux de recherche et développement à la croisée de l'agronomie, de la zootechnie et des sciences de gestion autour de concepts tels que le système fourrager (Duru $\&$ Hubert, 2003). Les exploitations d'élevage des grandes zones herbagères comme les moyennes montagnes d'Auvergne, où les prairies sont la base de l'alimentation des troupeaux au long des saisons, ont été particulièrement étudiées dans ce cadre.

Le maintien du pâturage dans ces systèmes de production de montagne est en effet porteur d'enjeux particuliers. Souvent présentée comme un "plateau de fromages ", l'Auvergne compte plusieurs Appellations d'Origine Protégée fromagères (AOP). Cette différenciation des produits préserve une dynamique laitière locale (Martin \& al., 2014). Lobligation de pâturage figure dans tous les cahiers des charges de ces AOP, comme par exemple celui de la Fourme d'Ambert qui stipule qu'« en période de disponibilité d'herbe, dès que les conditions climatiques le permettent, le pâturage des vaches laitières en lactation est obligatoire. Sa durée ne peut être inférieure à 150 jours par an » (Ministère de l'Agriculture, 2014). De plus, des études montrent que le pâturage des animaux est

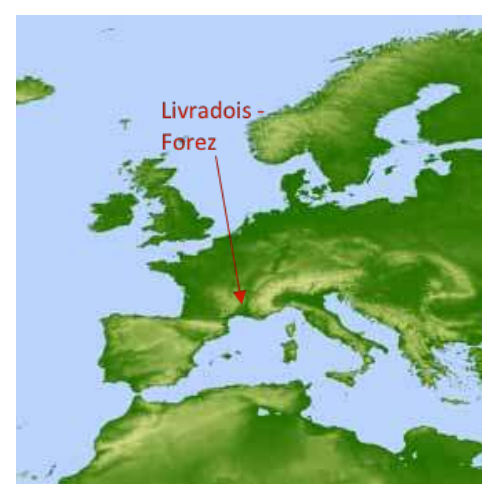




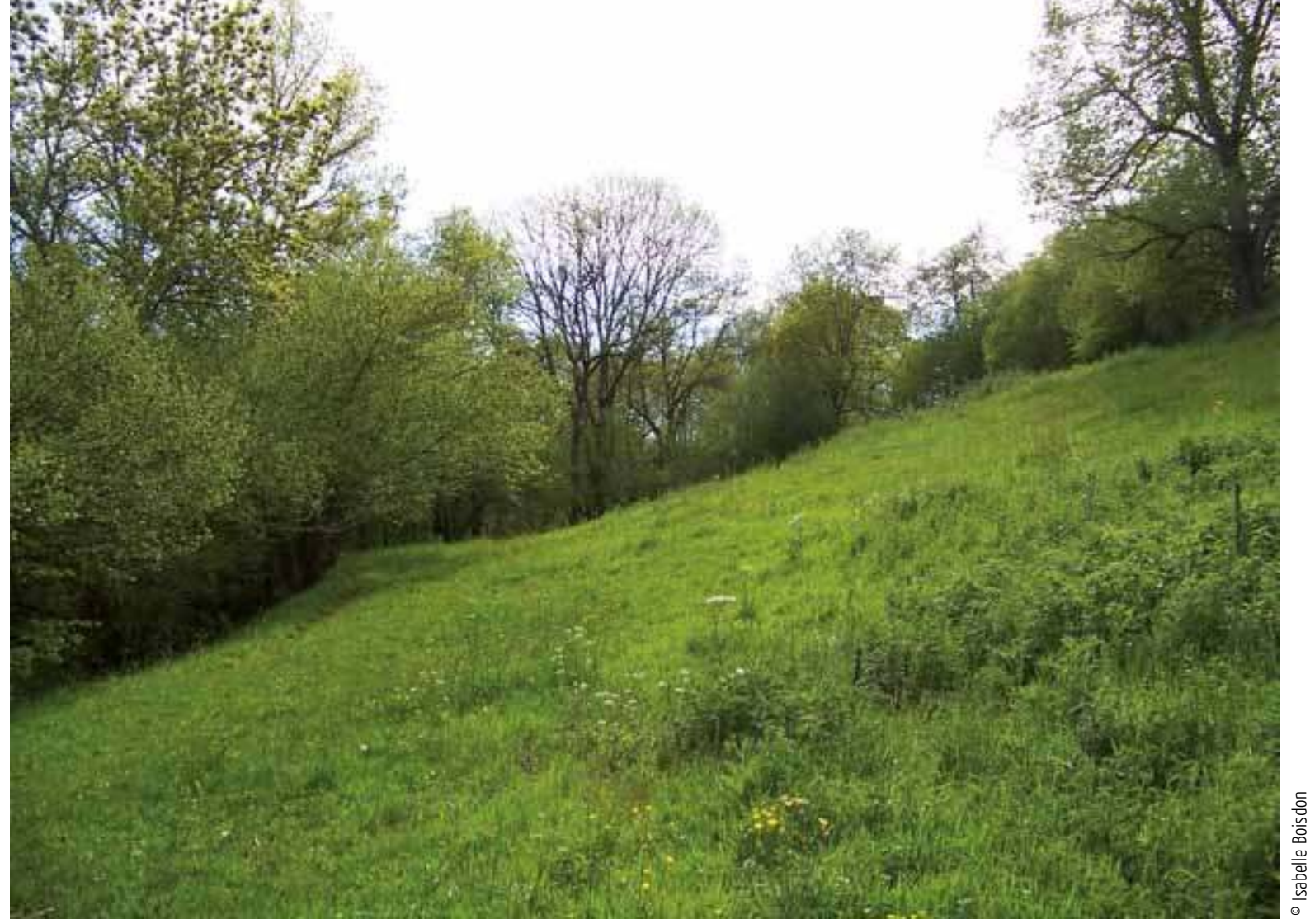

\section{Des parcelles avec de nombreuses contraintes}

« C'est mouillé, c'est pentu. C'est mal accessible en plus... Pour y accéder il faut descendre par un sentier

de bois », c'est ainsi que les éleveurs rencontrés qualifient certaines de leurs parcelles.

Le territoire de Condat-lès-Montboissier, comme celui du PNR Livradois-Forez, est fortement boisé

(55\% sont recouverts par la forêt). Un travail d'analyse du parcellaire communal nous a permis de mettre en évidence que $57 \%$ des parcelles agricoles étaient limitrophes d'une forêt et $96 \%$ situées à moins de 100 mètres d'une forêt (Mettez 2013). De nombreuses petites parcelles sont ainsi entourées

de bois, ce qui peut limiter leur accessibilité.

Des problèmes d'humidité des sols contraignent également l'usage des parcelles au printemps. Ceci

est fréquent sachant qu'une parcelle sur six est traversée par un cours d'eau ou une zone humide.

Assistant à la disparition progressive des exploitations dans la zone: « Quand vous regardez la production laitière quil y avait dans

les communes hautes, il n'y en a plus... », l'un des éleveurs rencontrés voit sa commune comme le dernier rempart contre la forêt: « A Condat, il y a [encore des exploitations]. Après, là-haut c'est fini... il y a que les sapins là-haut... [au-dessus de $900 \mathrm{~m}]$ ». La forêt est ici vue comme une menace pour l'agriculture, alors qu'au Brésil, notamment, la situation est opposée: c'est l'agriculture qui menace

la forêt (voir Poccard \& al. dans ce numéro). la méthode la moins coûteuse pour exploiter la ressource herbagère, le coût unitaire de l'herbe pâturée étant au minimum réduit de moitié par rapport à l'herbe récoltée (Réseaux d'Élevage Auvergne et Lozère, 2008). L'intérêt des prairies dépasse aussi la question alimentaire et le seul monde agricole. En effet, le développement durable de l'élevage dans les territoires, relayé et encouragé par les politiques publiques, est fortement conditionné par la présence des prairies et le maintien du pâturage, reconnaissant aux prairies pâturées des intérêts multiples: réservoir de biodiversité, lieu de stockage du carbone, sans oublier leur valeur esthétique pour le paysage (Huyghe, 2009). Les organismes de conseil aux agriculteurs ont de ce fait développé récemment des outils pour promouvoir et accompagner une organisation du pâturage qui valorise au mieux les divers atouts de la ressource fourragère (Peyraud \& Delaby, 2005 ; Moreau, 2014). Les préconisations des chercheurs et des techniciens sur la gestion du pâturage sont donc essentielles à plusieurs titres car du point de vue des institutions professionnelles, son organisation doit permettre, dans un contexte de renchérissement des intrants, de produire de la viande ou du lait à moindre coût tout en conservant un lien fort au territoire et en participant à la préservation de l'environnement. Mais qu'en est-il pour les éleveurs dans leur pratique quotidienne? Quelle place réservent-ils aux prairies et au pâturage dans leur exploitation? Comment perçoivent-ils cette ressource? La réponse n'est bien sûr pas unique car le pâturage ne se pratique pas d'une seule et même manière au sein d'un même territoire. L'objet de cet article est de montrer les disparités existantes entre les modalités de pâturage dans une même petite région, et d'en décrypter le sens du point de vue des stratégies d'éleveurs. Pour ce faire, nous prendrons appui sur deux situations contrastées observées dans une même commune que nous analyserons avec les outils de la zootechnie et de l'agronomie. Nous montrerons comment ces choix de pâturage sont 
directement liés à une diversité d'éléments passés et présents, à partir desquels chaque éleveur construit sa stratégie de production.

Notre analyse est développée dans le contexte particulier du Parc Naturel Régional (PNR) Livradois-Forez. Dans ce territoire de moyenne montagne situé à l'est du Massif central et encadré par Clermont-Ferrand, Saint-Etienne et Le-Puy-en-Velay, l'élevage bovin laitier et allaitant est une activité essentielle, largement basée sur l'exploitation des ressources herbagères qui représentent les deux tiers des surfaces agricoles. Celles-ci sont toutefois concurrencées par la forêt qui recouvre plus de $50 \%$ du territoire. L'élevage est ici primordial pour le maintien d'une dynamique rurale démographique et économique, et de l'équilibre fragile entre forêt, agriculture et autres activités. Il permet de limiter la fermeture du paysage et de préserver des emplois locaux, dans des contextes difficiles en termes de foncier (relief, altitude, morcellement, fragmentation et éloignement) et de démographie (vieillissement de la population) (Houdart $\&$ al., 2012).

Nous avons ainsi cherché à comprendre et expliciter la place du pâturage dans le fonctionnement des exploitations et les stratégies d'éleveurs associées en nous appuyant sur deux modalités extrêmes rencontrées dans une commune: des bêtes toujours dedans (zéro pâturage) ou toujours dehors dans un même pré (pâturage continu). La commune choisie pour cette étude est représentative du Livradois-Forez, et diversifiée tant au niveau des systèmes de production que des modes d'occupation des sols.

\section{Cadre d’analyse}

Afin d'étudier la place du pâturage dans le fonctionnement des exploitations, nous nous intéressons aux pratiques des agriculteurs et plus particulièrement celles relatives à l'utilisation des surfaces, pour analyser la justification de leur adoption par les éleveurs et les incidences agronomiques de ces choix (Gras \& al., 1988). Pour cela, considérant l'exploitation agricole comme un système (Bonneviale \& al., 1989), nous analysons ces pratiques à différents niveaux de cohérence. Tout d'abord au niveau du système de production, défini comme la combinaison des facteurs de production (terres, équipements, travail, ...) et des productions de l'exploitation agricole (Brossier, 1987). Ce concept permet de comprendre la place de l'élevage dans l'exploitation, le poids des structures de production et de leur évolution mais aussi du travail dans la place qui est faite au pâturage. Notre analyse se fait aussi au niveau du système d'élevage (Landais, 1994), ensemble des éléments organisés par l'homme en fonction de ses objectifs pour faire produire et se reproduire un collectif d'animaux domestiques en valorisant et renouvelant certaines ressources. La diversité des conduites que l'on peut observer dans un ensemble d'exploitations est en partie liée aux objectifs des éleveurs, et l'approche par le système d'élevage permet de rendre compte, dans leur globalité, des différents choix organisationnels qu'ils opèrent (Dedieu \& al., 2008). Ceci permet par exemple de décrire le fonctionnement des exploitations laitières à haute ambition productive rencontrées dans le Livradois, qui ont des logiques originales pour la zone. Enfin nous étudions les pratiques au niveau du système fourrager, concept élaboré afin de mettre en évidence l'existence d'interactions multiples entre surfaces et troupeaux (Duru \& al., 1988 ; Moreau \& al., 2009). Son rôle est central dans les exploitations d'élevage, où il permet de rendre compte de l'équilibre 


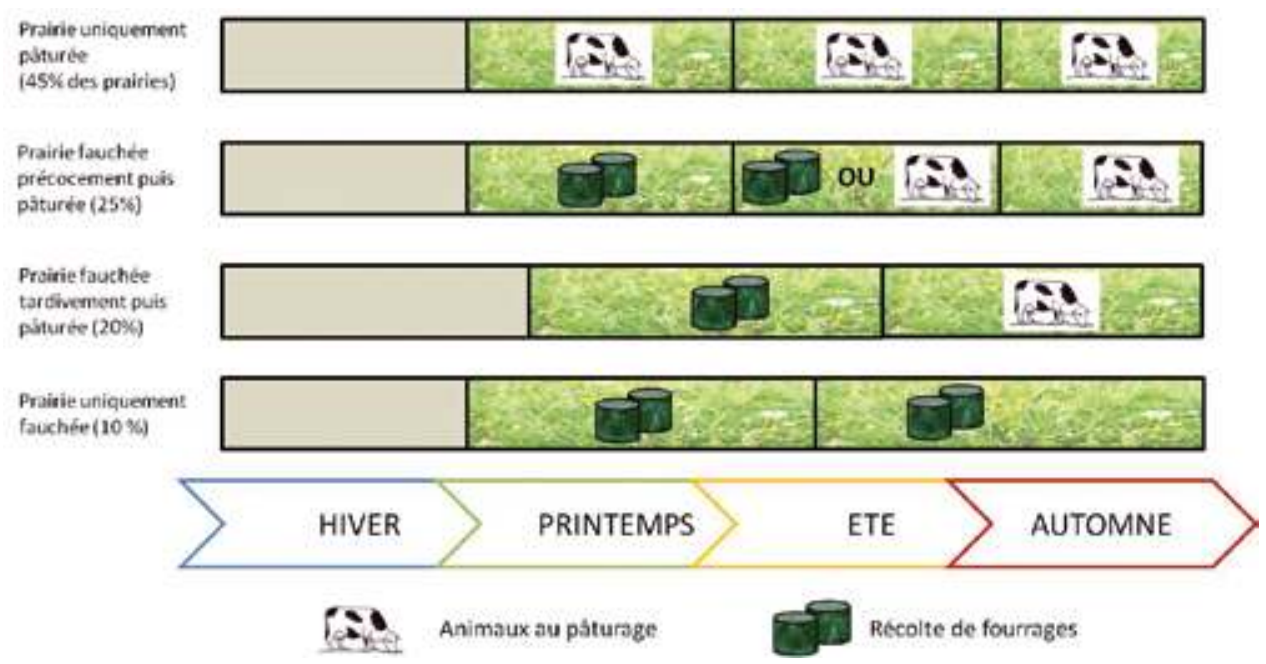

L'histoire d'une prairie au cours des saisons

Les différents types d'utilisation des prairies rencontrés en Auvergne sont présentés ici. Même si, au début du printemps, léleveur prédéfinit l'usage de chaque prairie, il peut être amené à modifier ses plans au fil de la saison. II est fréquent en effet qu'une parcelle prévue pour la pâture soit finalement fauchée quand la croissance de l'herbe a été plus rapide que la consommation du troupeau. A contrario une parcelle prévue pour la fauche peut être pâturée si la pousse de l'herbe sur les parcelles prévues pour le pâturage est insuffisante pour nourrir le troupeau. Des ajustements entre fauche et pâture sont ensuite possibles en été et à l'automne. Le " pilotage » au jour le jour des prairies est alors moins tendu pour l'éleveur, car la pousse d'herbe est moins rapide et moins importante. Cependant, en cas de mauvaises récoltes de printemps (saison froide, humide, ou au contraire sèche), l'éleveur pourra renforcer ses stocks par de nouvelles fauches. Enfin, en cas de sécheresse estivale, il peut distribuer des fourrages stockés à ses animaux. Il peut aussi être amené à acheter des fourrages à l'extérieur en cas de déficit important.

Source des pourcentages: SRISE Auvergne (2013) entre ressources fourragères et besoins des animaux, dans le respect des objectifs de l'éleveur et des contraintes propres à l'élevage. Au sein du système fourrager, la conduite du pâturage est un processus adaptatif qui s'articule au fil de l'année avec la constitution des stocks. À chaque étape l'éleveur prend en compte une série de facteurs. Ce sont des variables d'état concernant la qualité de la ressource fourragère et son évolution (qualité de l'herbe sur pied et du fourrage qui pourra être récolté, vitesse de pousse de l'herbe) mais aussi l'affectation des moyens (en main-d'œuvre ou financiers) à l'échelle du système de production. Ces déterminants jouent sur des horizons temporels différents (Duru \& Hubert 2003; Huyghe \& Delaby, 2013). Cela se traduit au niveau de l'exploitation par une diversité de conduite des prairies au cours d'une année avec alternances de phases de pâturage et de récolte d'herbe, qui définissent différents profils de parcelles. Nous verrons comment et en quoi, pour les éleveurs, la recherche d'un équilibre entre besoins du troupeau et ressources fourragères dans un objectif de production donné va influencer la place et l'organisation du pâturage dans leurs exploitations. Nous montrerons enfin que cet équilibre dépend d'un ensemble d'éléments présents et passés tels que le travail disponible, ou encore l'organisation spatiale de l'exploitation ou même l'histoire familiale.

\section{Des enquêtes en exploitation}

Les données sur lesquelles nous avons travaillé sont issues d'enquêtes menées auprès d'agriculteurs sous la forme d'entretiens semi directifs, au cours desquels nous leur avons demandé de parler de leurs choix en termes de pâturage (Darré \& al., 2004). Ces entretiens étaient donc centrés sur les pratiques relatives à l'utilisation des surfaces. Sur la base d'une représentation cartographique du territoire, nous avons demandé aux agriculteurs de décrire leurs parcelles et d'expliciter ce qu'ils réalisent sur chacune d'elles. Les entretiens ont été partiellement retranscrits, et les logiques d'action des éleveurs (Begon $\&$ al., 2009) ont été identifiées pour chacune des exploitations. Les deux cas que nous présentons correspondent à des modalités extrêmes d'organisation du pâturage qui nous permettent d'identifier les éléments majeurs structurants de la diversité des stratégies de conduite du pâturage. Le premier (le zéro pâturage) se rencontre dans deux systèmes 
laitiers qui excluent le pâturage pour mieux gérer l'herbe et le troupeau et le deuxième (le pâturage continu) chez un éleveur allaitant où le pâturage est pensé pour limiter au maximum le travail de surveillance et de conduite des animaux. Le zéro pâturage est un mode d'alimentation dans lequel les animaux productifs n'ont pas d'accès à un pâturage et restent toute l'année à l'étable. Le fourrage, coupé mécaniquement, leur est distribué frais ou sous forme conservée. Dans le cas du pâturage continu, les animaux consomment l'herbe disponible sur une parcelle unique, de grande taille. Des fourrages complémentaires peuvent être distribués en cas de pousse insuffisante de l'herbe.

\section{La place du pâturage dans le système de production}

Les éleveurs laitiers pratiquant le zéro pâturage ont choisi de s'agrandir afin de produire d'importantes quantités de lait. Les deux exploitations concernées par cette organisation du pâturage sont des structures de grandes dimensions pour le Livradois-Forez, et même pour la France. Elles exploitent presque un tiers des surfaces agricoles de la commune soit plus de 100 ha chacune avec un troupeau de 80 à 120 vaches, et leur production laitière est de 700000 à 800000 litres par an. En 2012 la France comptait 3300 élevages laitiers de plus de 100 vaches, soit $4 \%$ des exploitations laitières du pays (Perrot, 2012). La majeure partie de ces grandes exploitations laitières (au sens de Beguin \& Roussel, 2006) sont localisées en plaine ( 84 \% d'entre elles se trouvent en régions de plaine de la moitié nord de la France). De telles structures sont donc atypiques dans une zone de moyenne montagne comme le Livradois-Forez. Le premier éleveur que nous avons rencontré (Bernard, 61 ans) est associé avec ses deux fils. Il a atteint l'âge de la retraite mais reste dans la société tant que cela est possible afin de conserver des droits à primes. Le second (Olivier, 45 ans) est associé avec un autre éleveur, et réfléchit à l'installation prochaine de son fils. Leurs exploitations se sont agrandies lors d'installations successives des différents associés. Malgré un effort de concertation entre éleveurs intéressés par des surfaces dans la commune, l'extension n'a pas pu se faire à proximité du siège de l'exploitation et le parcellaire obtenu est resté très dispersé. Ils ont alors réalisé le choix de déplacer l'herbe plutôt que le troupeau de vaches laitières. En effet, la traite est une contrainte qui pèse lourd, les vaches laitières en production devant rejoindre la

\section{Organisation du parcellaire des exploitations en zéro pâturage} Les surfaces de ces exploitations sont situées sur au moins quatre communes différentes et 9 sites distants d'au moins un kilomètre. Près de $80 \%$ des parcelles sont situées à plus d'un kilomètre du bâtiment principal des vaches laitières. Ce parcellaire dispersé est perçu comme une contrainte par les éleveurs. Se comparant à des éleveurs d'autres zones disposant d'un parcellaire plus groupé, ils expriment: « lls ne savent pas ce quilis ont ces gens, ils ont de l'or par rapport à nous, par rapport à notre parcellaire [...], vous vous rendez compte? Moi je dis, c'est un rêve ça, c'est un rêve... d'avoir un domaine tout autour de ses bâtiments... ils ne se rendent pas compte les gens qui ont ça ». Du fait de cette dispersion, l'intervention sur les parcelles est plus longue, car il faut d'abord y accéder.

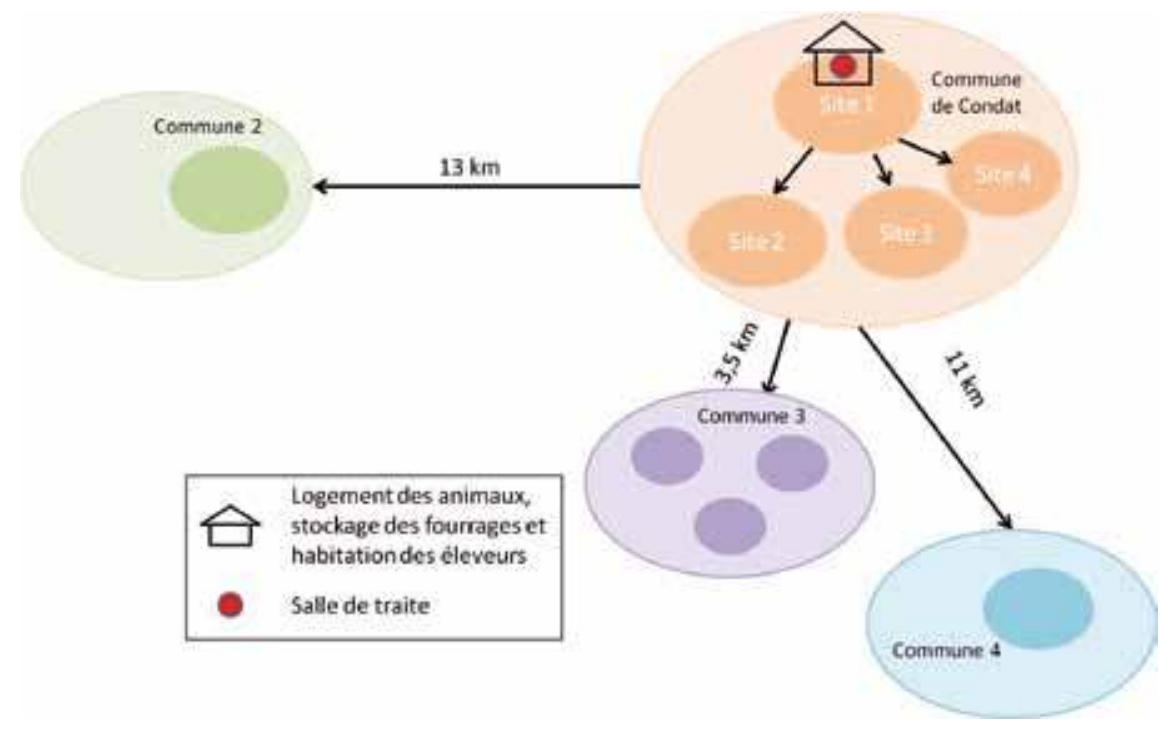


Des contraintes de pente, d'humidité, d'accès de certaines parcelles aux engins agricoles, expliquent donc la présence des vaches allaitantes sur ces parcelles aux potentialités limitées. Sur cette exploitation les ressources herbagères sont constituées uniquement de prairies permanentes, et contrairement au premier cas l'ensemble des parcelles se trouve sur la commune. L'objectif de l'éleveur est avant tout de se libérer du temps pour le consacrer aux cultures. Grâce au pâturage continu il ne passe pas de temps à déplacer son troupeau d'une parcelle à une autre. De plus, il limite volontairement le nombre de vaches de son troupeau sinon, comme il le formule: "Ça ne passerait pas, il faudrait que je les change de pré ».

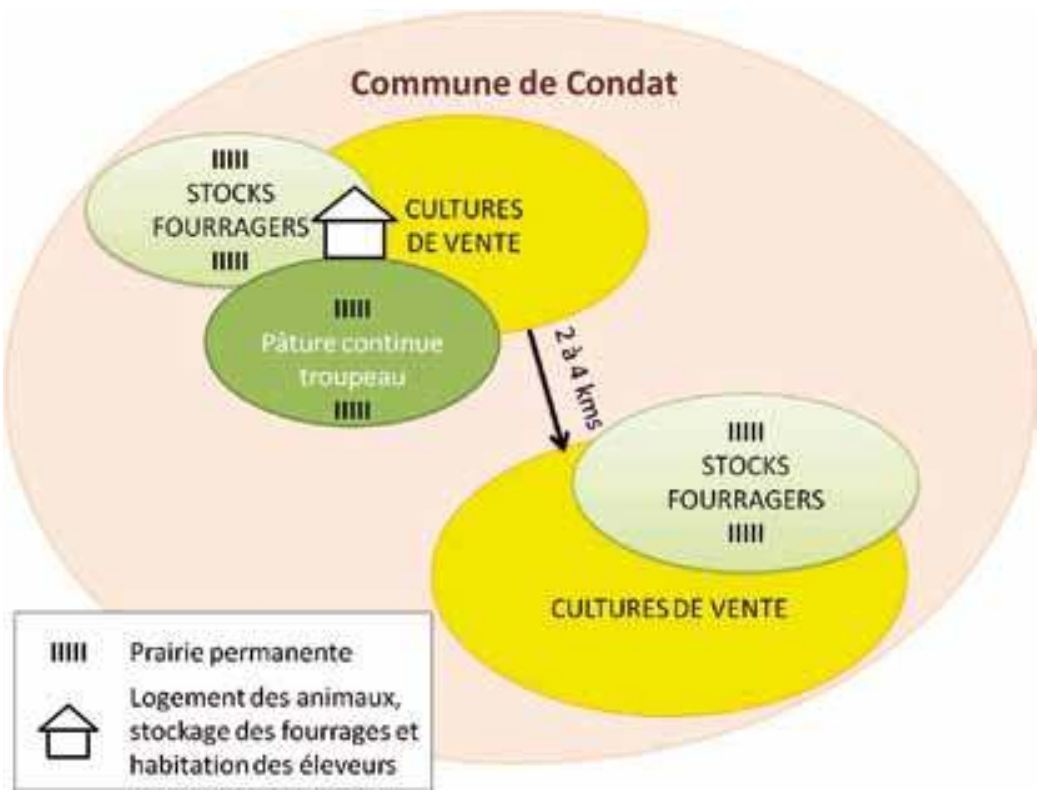

Le parcellaire de l'exploitation en pâturage continu et son utilisation

Les parcelles labourables sont consacrées aux cultures, les autres restent en prairies permanentes, pour la pâture ou pour la réalisation de stocks. L'assolement des cultures est prévu de manière à limiter les contraintes dues au morcellement en regroupant dans une même zone des parcelles ayant le même type de couvert. 


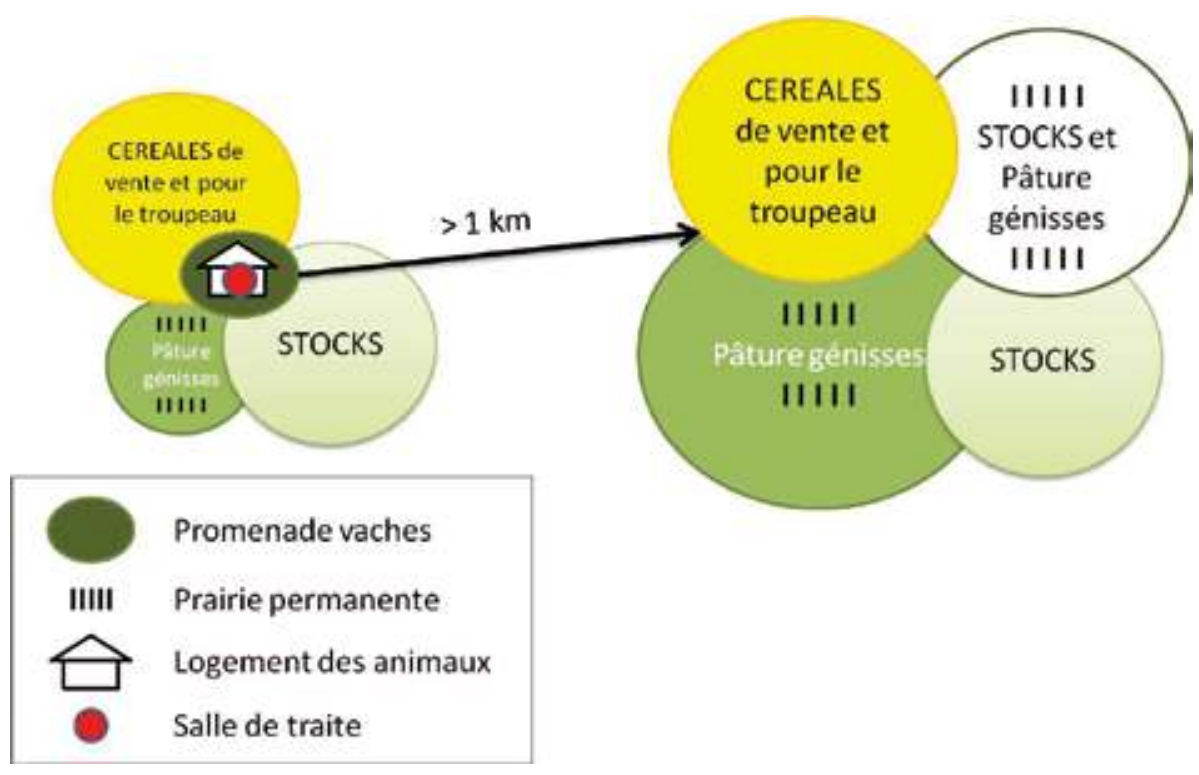

Cependant l'éloignement et le morcellement des parcelles compliquent les déplacements d'animaux qui doivent, parfois, être réalisés à l'aide d'une bétaillère. Cette organisation est contraignante, comme le rappelle Olivier: «Chaque fois qu'on veut déplacer... comme hier, on a commencé à lâcher des animaux et que je te prends la bétaillère et que je t'amène... ».

Dans le cas de l'éleveur allaitant la conduite du troupeau est pensée pour être simple et ne pas prendre de temps, afin d'en libérer pour les opérations chronophages liées à la production de plants de pomme de terre (épuration ${ }^{1}$, arrachage et calibrage), qui lui plaît et est sa principale source de revenu. Tous les bovins sont conduits ensemble en un seul lot, regroupant les vaches, les veaux, le taureau, et les génisses de tous âges. La reproduction n'étant pas contrôlée par l'éleveur, des naissances ont lieu toute l'année. Pour limiter le nombre de manipulations les ventes regroupent des animaux d'âges différents (de 8 à 14 mois). Le suivi quotidien du troupeau est également simplifié. Tout au long de l'année les animaux ont librement accès à un bâtiment ouvert en permanence ainsi qu'à un râtelier libre-service avec du foin l'hiver et de la paille l'été, et « [Les vaches] font ce qu'elles veulent ». Contrairement à l'autre cas, cette exploitation ne met pas en avant des ambitions productives sur l'atelier bovin ni sur les surfaces qui lui sont dédiées. Les performances zootechniques du troupeau arrivent en second plan dans les préoccupations de l'éleveur, derrière les performances des cultures. La conduite non contrainte du troupeau étant associée à une utilisation extensive de la ressource, les performances animales se déduisent des ressources fourragères disponibles.

\section{La place du pâturage dans le système fourrager}

Dans le cas du zéro pâturage, le système est conçu pour assurer une forte productivité des vaches laitières et s'appuie avant tout sur les parcelles labourables, donc sur des cultures fourragères pour assurer l'alimentation des animaux en quantité comme en qualité. Maïs et céréales sont implantés sur les meilleures parcelles, y compris à proximité des bâtiments, et alternent avec de l'herbe cultivée qui nécessite du soin. L'enchaînement entre couverts est très rapide pour ne pas perdre de temps entre deux cultures et produire de grandes quantités de fourrages. Pour gérer la distance entre le siège de l'exploitation et les parcelles éloignées, l'herbe est récoltée mécaniquement pour être distribuée soit en vert soit sous une forme 
conservée (ensilage ou foin). Complémentaire à cette recherche de volumes, Bernard comme Olivier visent aussi la qualité des fourrages proposés. Ils portent leur attention sur la culture du maïs, sur les espèces et variétés prairiales semées dans les prairies temporaires ainsi que sur les stades de récoltes de façon à stocker une herbe jeune et riche. Le système en zéro pâturage aboutit donc à un système technique complexe, avec un haut niveau d'intensification des pratiques sur les surfaces fourragères. Ces exploitants ont choisi de produire de gros volumes de lait en étant dégagés des contraintes imposées par la filière AOP. Ils n'ont donc pas d'obligation sur la conduite, l'alimentation ou le temps de pâturage des vaches laitières.

Dans le cas du pâturage continu, l'éleveur a organisé le pâturage dans un objectif de simplification. L'herbe est pâturée sur une seule parcelle par le troupeau allaitant constitué de l'ensemble des catégories d'animaux rassemblées en un seul lot. La seule intervention sur ce pâturage libre concerne la mise en défens d'une partie de la parcelle durant trois semaines, au printemps, une fois la majorité des mises bas effectuée, afin de surveiller plus facilement les veaux, faciliter la repousse de l'herbe et éviter le gaspillage dû au piétinement. Les récoltes de fourrages (exclusivement du foin) ont lieu en juin et septembre en dehors de périodes de travaux sur cultures. Toutes les parcelles destinées au troupeau sont affectées soit uniquement à la fauche soit uniquement à la pâture. Le temps consacré au pâturage est réduit et le travail se compose de peu de tâches (complémenter les animaux en foin, surveiller le troupeau). Les productions végétales sont prioritaires dans la planification des activités. Les foins de début juin précèdent les moissons et la période de travail intense dédiée aux plants de pommes de terre. Ils permettent d'assurer la quantité des stocks, mais leur qualité reste dépendante de l'année climatique et du stade de l'herbe au moment où l'éleveur est disponible pour la récolter. La repousse attend ensuite la fin de la récolte des plants de pommes de terre pour être fauchée fin août. Les soins aux parcelles de prairies permanentes sur lesquelles sont réalisés les stocks demandent peu de temps.

\section{Un bâtiment ouvert sur les pâtures, des bois qui servent d'abris}

Toute l'année les animaux ont accès à une unique parcelle de pâturage de 9 ha, attenante au bâtiment, avec des parties boisées pouvant servir d'abris. Ainsi l'agriculteur ne déplace pas son troupeau, il ne passe pas de temps à le changer de parcelle et ne doit pas non plus entretenir de clôtures sur l'ensemble de ses prairies: « Je dois être le seul à ne plus trimballer les vaches [...] C'est un peu de l'élevage spécial ».

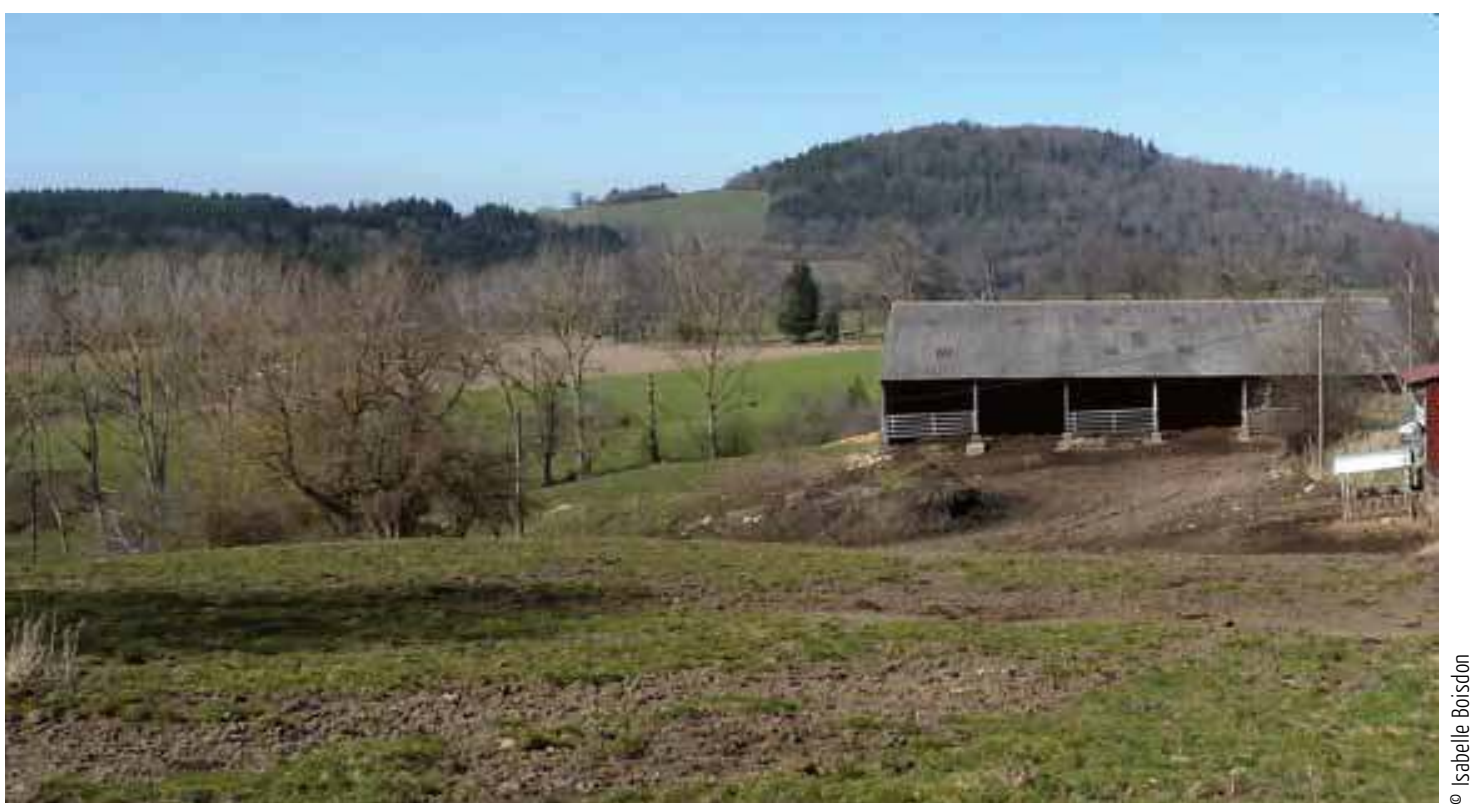




\section{Discussion}

Alors que les potentialités du milieu sont comparables pour les systèmes étudiés, leur organisation, dont celle du pâturage, prend des formes différentes selon leurs choix de production et leurs contraintes. Les exemples présentés nous montrent que l'agrandissement des structures et l'augmentation de la charge de travail des éleveurs les amènent à repenser leurs manières d'exploiter les surfaces. Nous nous sommes particulièrement attachés à décrire les déterminants internes aux exploitations qui prennent le pas sur des incitations extérieures comme les cahiers des charges des AOP ou les préconisations des chercheurs et techniciens et expliquent les pratiques de pâturages observées. Dans ce même numéro Napoléone \& al. décrivent, dans d'autres montagnes françaises, des ressorts de ces évolutions portées par l'élaboration, dans des collectifs professionnels, des normes de l'action qui convient en lien avec des appellations d'origines fromagères. Une analyse plus approfondie des conceptions des agriculteurs permettrait sans doute de rendre compte de l'imbrication entre ces deux dimensions pour comprendre la diversité des pratiques de mise en valeur de l'herbe par les éleveurs.

\section{Situation des animaux et mode d'alimentation au long de l'année selon les cas rencontrés}

Dans les deux cas rencontrés, il n'y a pas de déplacement des animaux d'une parcelle pâturée à une autre. Les animaux sont soit toujours à l'intérieur, soit toujours présents sur la même parcelle. Des fourrages stockés sont également distribués toute l'année aux animaux dans les deux cas.

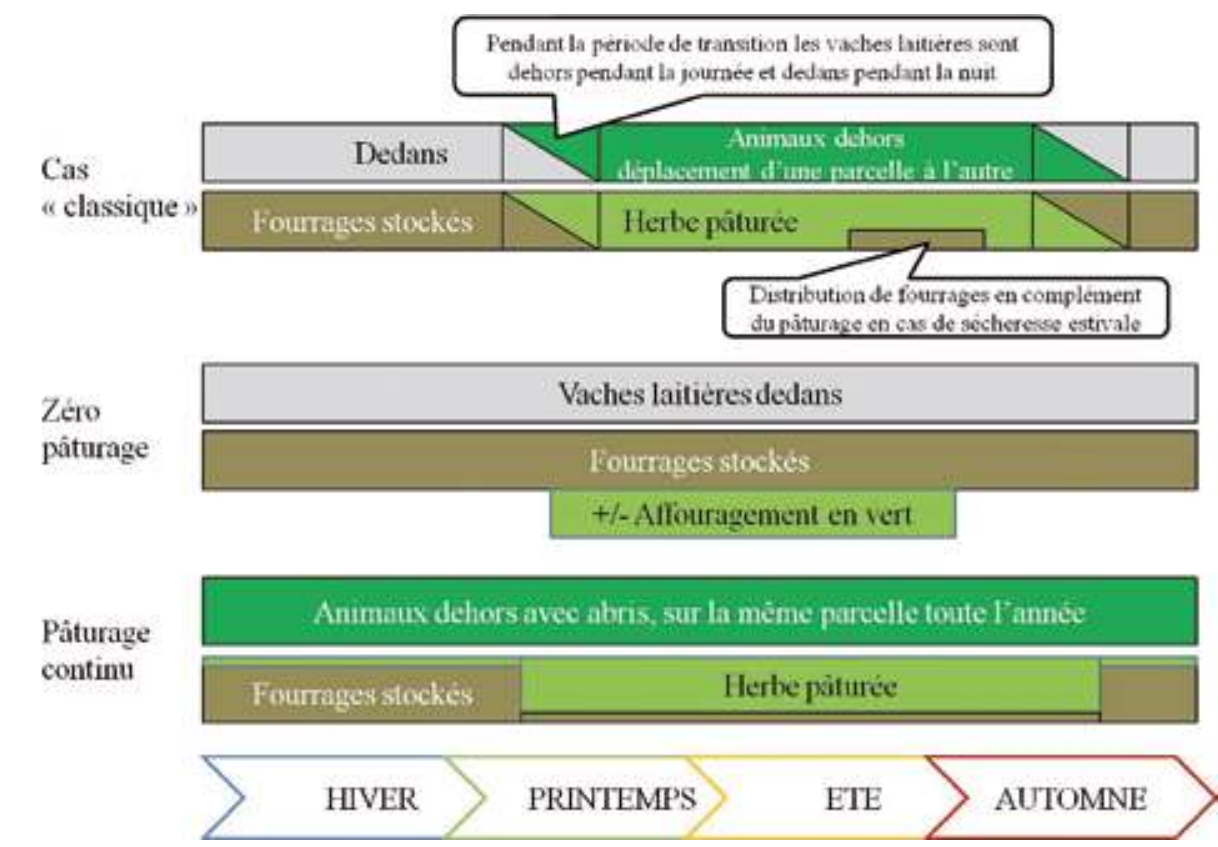

Dans les deux cas présentés, l'organisation classique du pâturage, avec des lots d'animaux qui se déplacent sur les parcelles, est perçue comme trop contraignante par les éleveurs qui recherchent une conduite d'élevage ne prenant pas trop de temps. Cette organisation est donc abandonnée au profit de systèmes originaux qui conviennent mieux aux éleveurs. Dans le premier cas, le travail avec les animaux est réalisé à l'intérieur des bâtiments. Seuls les tracteurs se déplaçant sur les routes, les chemins ou les champs 
sont perceptibles. Dans le second cas ce sont des animaux qui habitent en permanence ce paysage et qui sont visibles, prélevant sur place la ressource dont ils ont besoin. Ceci montre aussi combien les activités liées à la mobilisation des ressources herbagères peuvent marquer le paysage.

Malgré des modes d'alimentation des animaux qui diffèrent, nous observons des similitudes dans les modes d'organisation du pâturage dans les deux cas étudiés. Le point le plus visible est la séparation spatiale entre parcelles dédiées au pâturage et parcelles dédiées aux stocks fourragers. Du fait de la stabilité de l'alimentation des animaux tout au long de l'année, il y a aussi moins de période de transition alimentaire dans ces élevages; c'est un point que soulignent les éleveurs quand ils se situent par rapport aux préconisations qui leur sont adressées. Ils s'affranchissent ainsi de la gestion parfois délicate de ces périodes, soit pour mieux maîtriser leur production et atteindre un objectif de performance technique ambitieux, soit pour libérer du temps pour un autre atelier de l'exploitation. L'alternance fauche-pâture prônée par les agronomes pour une bonne exploitation des potentialités des prairies (GNIS, Peyre $\&$ al., 2009, Réseau Agriculture Durable) n'est pas donc pratiquée dans ces exploitations.

Les éleveurs présentés ont en outre choisi de reléguer les prairies permanentes au second rang derrière des cultures plus productives ou rémunératrices, sans adaptation de leur conduite à leurs potentialités propres. Les éleveurs laitiers ne misent pas sur la valorisation des prairies permanentes pour l'alimentation des animaux, mais portent leurs efforts sur des cultures fourragères et des cultures de vente, là où, agronomiquement parlant, le milieu est le plus favorable. Les cultures fourragères (maïs ensilage et prairies temporaires) représentent alors la plus grande part du volume de fourrages produits, ce sont elles qui font ressource pour l'alimentation des troupeaux. Les prairies permanentes sont moins fertilisées que les prairies temporaires et moins sollicitées du fait d'une moins grande attente des éleveurs vis-à-vis de la production de fourrages sur ces surfaces. Chez l'éleveur allaitant les prairies permanentes sont soit pâturées en continu, soit uniquement fauchées. Aucune fumure organique n'est apportée, l'éleveur gardant les faibles quantités d'effluents d'élevage produits pour les parcelles de cultures qui lui rapportent le plus. Alors que le potentiel de production de certaines prairies permanentes est reconnu (Huyghe 2008), nous montrons ici qu'elles peuvent prendre une place secondaire dans le système de production, même dans une zone de moyenne montagne où leur image est intimement liée aux productions sous signe de qualité.

Notre approche systémique des pratiques des éleveurs a montré sa pertinence pour la compréhension de situations contrastées d'insertion du pâturage dans le fonctionnement des exploitations. Elle mériterait certainement d'être complétée par un regard plus sociologique sur le lien au modèle d'élevage qui convient (voir Doré \& al. dans ce numéro) ou les raisons des logiques fourragères (Frezel 2010).

\section{$\&$}

Observer des pratiques de pâturage extrêmes et s'interroger sur leur sens et la diversité des éléments auxquels elles renvoient nous a permis de mieux comprendre comment des 
éleveurs s'adaptent à leur territoire tout en poursuivant leurs objectifs de production. Des facteurs aussi bien personnels qu'historiques ou liés au milieu expliquent les modes de pâturage rencontrés. Ces questions sont prégnantes pour les acteurs du développement agricole, dans le contexte actuel de questionnement sur les transformations des exploitations et l'accompagnement des agriculteurs. Ainsi, un regard porté uniquement sur le pâturage ou les prairies et oubliant la globalité du système, risque de passer à côté de facteurs d'adaptation des exploitations d'une autre nature.

Aujourd'hui les éleveurs que nous avons rencontrés doivent composer avec l'incertitude de la conjoncture agricole et du devenir des entreprises de collecte et de transformation des produits. Quelle peut être la place des systèmes particuliers présentés? La notion de complémentarité entre systèmes à l'échelle du territoire émerge en Livradois-Forez et est reconnue par les acteurs locaux. La diversité des systèmes présents sur la zone (orientation lait ou viande, petites ou grandes exploitations, éleveurs engagés dans les filières de qualité rémunératrices ou fournisseurs de produits standards) est nécessaire au fonctionnement des entreprises d'aval qui valorisent les produits, ce qui permet de pérenniser l'ensemble des modèles. L'enjeu de maintenir cette diversité est d'autant plus crucial que dans ce territoire, l'agriculture participe au maintien d'espaces ouverts en limitant l'extension de la forêt, mais également à celui des dynamiques rurale, démographique et économique.

Ce travail a été conduit dans le cadre du projet de recherche MOUVE financé par l'Agence Nationale de la Recherche (projet ANR-2010-STRA-005-01).

Auteurs (UMR1273 Metafort) : Isabelle Boisdon (VetAgro Sup, isabelle.boisdon@vetagrosup.fr), Claire Balay (VetAgro Sup,claire.balay@vetagro-sup.fr), Jean-Yves Pailleux (INRA, pailleux@clermont.inra.fr),Nathalie Hostiou (INRA,nhostiou@clermont.inra.fr),Hélène Rapey (IRSTEA, helene.rapey@irstea.fr),Sylvie Cournut (VetAgro Sup,sylvie.cournut@vetagro-sup.fr). 


\section{NOTES}

Photo d'ouverture :

La récolte mécanique des fourrages remplace le pâturage. Dans les exploitations en zéro pâturage, les animaux productifs restent dans les bâtiments, les hommes se déplacent de parcelle en parcelle sur des tracteurs pour récolter les fourrages nécessaires à leur alimentation.

1. Epurer consiste à enlever du champ les plantes malades, afin de respecter les règles de production des semences certifiées (moins de $3 \%$ de plants malades). Cela se fait à partir du 10 au 15 juin et pendant 1 mois environ. Il faut pour cela

passer dans les champs au lever du jour pour repérer les plantes malades, les arracher et les sortir de la parcelle pour les détruire. 2 à 3 passages sont nécessaires en fonction du nombre de plantes malades repérées au premier passage.

\section{POUR CITER CET ARTICLE}

Boisdon, I., Balay, C., Pailleux, J.-Y. \& al. 2015 Pâturer: regards agronomiques et pratiques d'éleveurs. In Dupré, L., Lasseur, J. \& R. Poccard-Chapuis Pâturages TechniquesÉCulture 63: 38-55 


\section{RÉFÉRENCES}

Begon, M., Pailleux, J.-Y., Joly, N., \& al. 2009 Les Chemins pour durer en élevage bovin laitier: diversité des logiques d'action sur le long terme en Ségala (Massif Central), Rencontres Recherches Ruminants 16: 105-108.

Beguin, E. et Roussel P. 2006 Les Grandes exploitations laitières en France : état des lieux, fonctionnement, performances techniques et économiques, Rencontres Recherches Ruminants 13: 353-356.

Bonneviale, J.-R., Jussiau, R., Marshall, E. 1989 Approche globale de l'exploitation agricole; comprendre le fonctionnement de l'exploitation agricole: une méthode pour la formation et le développement. Dijon: INRAP.

Brossier, J. 1987 Système et système de production. Note sur ces concepts, Cahiers des Sciences Humaines 23 (3-4): 377-390.

Darré, J.-P., Hubert, B., Landais, E., Lasseur J. 1993 Raisons et pratiques. Dialogue avec un éleveur ovin, Études Rurales 131-132: 153-163.

Darré, J.-P., Mathieu, A., Lasseur, J. (Dir.) 2004 Le Sens des pratiques: conceptions d'agriculteurs et modèles d'agronomes. Paris: INRA Editions.

Dedieu, B., Faverdin, P., Dourmad, J.-Y., \& al. 2008 Système d'élevage, un concept pour raisonner les transformations de l'élevage, INRA Productions Animales 21 (1): 45-58.

Duru, M., Nocquet, J., Bourgeois, A. 1988 Le système fourrager: un concept opératoire? Fourrages 115: 251-272.

Duru, M. et Hubert, B. 2003 Management of grazing systems: from decision and biophysical models to principles for action, Agronomie 23: 689-703.

Frezel, J. 2010 De la conception du métier aux pratiques: Le système fourrager chez les éleveurs bovins laitiers du Grand Ouest. Toulouse: INP ENSAT (mémoire de fin d'études ingénieur, DAA Système de production, environnement et territoire).

GNIS Le diagnostic des prairies. Les méthodes d'amélioration par la conduite, [en ligne] URL: http://www.prairiesgnis.org/pages/methodes.htm. Consulté le 20 mai 2014.

Gras, R., Benoit, M., Deffontaines, J.-P., \& al. 1988 Le Fait technique en agronomie. Activité agricole, concepts et méthodes d'étude. Paris: INRA et L'Harmattan (Alternatives Rurales).

Houdart, M., Cournut, S., Rapey, H., \& al. 2012 La Contribution de lélevage au développement durable des territoires: point de vue d'acteurs du territoire du Livradois-Forez, Auvergne. Clermont-Ferrand: IUFM éditeur (Colloque des 19 au 20 décembre 2012: Les représentations Nord-Sud du développement durable IUFM d'Auvergne).

Huyghes, C. 2008 La Multifonctionnalité des prairies en France: I. Les fonctions de production, Cahiers Agricultures 17 (5): 427-435.

- 2009 La Multifonctionnalité des prairies en France: II. Conciliation des fonctions de production et de préservation de l'environnement, Cahiers Agricultures 18 (1): 7-16.

Huyghes, C. et Delaby, L. 2013 Prairies et systèmes fourragers. Pâturage - Ensilage - Foin. Paris: Éditions France Agricole (Agri Production).

Landais, E. 1994 Système d'élevage. D'une intuition holiste à une méthode de recherche, le cheminement d'un concept. In C. Blanc-Pamard \& J. Boutrais (Dir.) Dynamique des systèmes agraires: à la croisée des parcours. Paris: ORSTOM: 15-49.

Mathieu, A., 2004 Conceptions des agriculteurs et modèles agronomiques. Le pâturage des vaches laitières dans le Jura, Natures Sciences Sociétés 4 (12): 387- 399.

Martin, B., Lherm, M, Béranger, C. 2014 Évolutions et perspectives de l'élevage des ruminants dans les montagnes françaises, INRA Productions Animales 27 (1): 5-16.

Mettez, C. 2013 Appréciation des services éco-systémiques liés à l'activité d'élevage: Test d’une méthode sur Condatlès-Montboissier. Clermont-Ferrand: IADT (mémoire de Master 1 Développement des Territoires et Nouvelles Ruralités). 
Ministère de l'Agriculture, de l'Agroalimentaire et de la Forêt, 2014 Cahier des charges de l'appellation d'origine protégée «Fourme d'Ambert » associé à l'avis n`AGRT1413717V. Paris: JORF (Bulletin officiel du Ministère de l'agriculture, de l'agroalimentaire et de la forêt n²7-2014).

Moreau, J.-C., Delaby, L., Duru, M., \& al. 2009 Démarches et outils de conseil autour du système fourrager: évolutions et concepts, Fourrages 200 : 565-586.

Moreau, J.-C. (Dir.) 2014 Organiser le pâturage et gérer le parcellaire. Démarche de conseil nº 5 élaborée dans le cadre du CASDAR PraiCoS. Paris: Institut de l'Élevage (Guide Méthodologique).

Perrot, C. 2012 France laitière 2015 : la recomposition territoriale est déjà en marche! Rennes: AgroCampus Ouest (Journée CEREL du 28 juin 2012, Vers une concentration territoriale de la production laitière en France après 2015).

Peyraud, J.-L. et Delaby, L. 2005 Combiner la gestion optimale du pâturage et les performances des vaches laitières: enjeux et outils, INRA Productions Animales, 18 (4): 231-240.

Peyre, G., Coulaud, F., Dampfhoffer, M., \& al.2009 Croiser les approches agronomiques et anthropologiques pour mieux appréhender les capacités d'adaptation des systèmes d'élevage face aux pullulations de campagnols, Rencontres Recherche Ruminants 16: 97-100.

Réseau Agriculture Durable, 2011 Entretenir ses prairies pour qu'elles durent, [en ligne] URL: http://www. agriculture-durable.org/wp-content/uploads/2011/05/EntretenirSesPrairiesBAT.pdf. Consulté le 20 mai 2014.

Réseaux d'Élevage Auvergne et Lozère, 2008 Coût des fourrages 2008. Prairies, maïs ensilage et céréales à paille. Clermont-Ferrand: Chambres d'Agriculture et Institut de l'Élevage (Références). 


\section{RÉSUMÉ}

Pâturer: regards agronomiques et pratiques d'éleveurs. La conduite du pâturage comme révélateur des stratégies d'éleveurs dans le Livradois-Forez. Depuis les années soixante, l'amélioration des rations alimentaires des animaux a fortement contribué aux accroissements de productivité dans les élevages. Lorsque les fourrages pâturés sont une composante significative des rations, notamment dans les régions où le climat varie fortement entre les saisons, cette amélioration implique de gérer des équilibres complexes et en « temps réel » entre les disponibilités en fourrage et les besoins des animaux. De plus, l'éleveur doit intégrer dans ses décisions des contraintes du milieu variées, des éléments réglementaires liés à la préservation de l'environnement et des injonctions venant des filières commercialisant ses produits. Les moyennes montagnes d'Auvergne, où les prairies sont la base de l'alimentation des troupeaux au long des saisons, sont un bon support pour s'intéresser à ces questions. Le pâturage est ici porteur d'enjeux particuliers, et comprendre les modes de gestion de l'alimentation au pâturage nécessite un regard appuyé sur les conceptions et les pratiques des agriculteurs.

Cet article s'intéresse à deux modalités de pâturage particulières et contrastées, observées dans une commune du Livradois-Forez: des bovins toujours dedans ou toujours dans le même pré. Entre zéro pâturage et pâturage continu, il montre, à l'aide du cadre d'analyse agronomique, en quoi la place accordée au pâturage reflète des stratégies d'éleveurs qui ne se limitent pas à la seule finalité d'alimenter des animaux, et dépendent à la fois de facteurs naturels, historiques et humains. Ainsi de grandes exploitations laitières ont choisi le zéro pâturage pour gérer plus facilement leur parcellaire morcelé, préserver la ressource fourragère et avoir une ration stable et de qualité. Un autre éleveur a choisi le pâturage continu pour se libérer du temps pour un atelier végétal rémunérateur. Ces réalités interrogent les modèles agricoles et les modes d'accompagnement des éleveurs, qui doivent prendre en compte que la diversité des systèmes est peut être une condition du maintien de l'élevage dans ces zones de moyenne montagne

\section{ABSTRACT}

Grazing management, an indicator of livestock farmers' strategies in the Livradois-Forez. Since the 1960s, the improvement of animal feed rations has greatly contributed to the increase in productivity on livestock farms. When grazed forage is a significant component of animal feed rations, especially in regions where the climate varies considerably between seasons, this improvement involves the management of complex balances, in real time, between forage availability and the animals' needs. Moreover, the livestock farmer must take the various environmental limitations, regulations linked to the preservation of the environment, and injunctions from the sectors that market his products into account in his decisions. The medium mountains of Auvergne where grassland constitutes the feed base for the herds throughout the seasons are a good case study to address these questions. Grasslands here imply specific challenges, and to understand the different ways of managing grassland feed requires an in-depth look at the approaches and practices of livestock farmers.

This article focuses on two specific and contrasted grazing systems observed in the Livradois-Forez region: bovines always inside or always in the same meadow. Between zero grazing and continuous grazing, it is shown, using an agronomic analysis framework, how the degree of importance attributed to grazing reflects livestock farmers' strategies that are not limited to feeding the animals alone, but depend on natural, historical and human factors as well. Thus, large dairy farms have chosen zero grazing to more easily manage their fragmented parcels, to preserve their forage resource and to have a stable and high-quality feed ration. Another livestock farmer has chosen continuous grazing to free up time for a more lucrative crop production unit. These different realities question agricultural models and ways in which to help livestock farmers, which must take account of the fact that the diversity of systems is perhaps a condition for maintaining livestock breeding in these middle mountain regions. 


\section{MOTS-CLÉS}

Livradois-Forez, prairies, élevage ruminant, pratiques, alimentation animale

\section{KEYWORDS}

Livradois-Forez, grasslands, ruminant livestock breeding, practices, animal feed

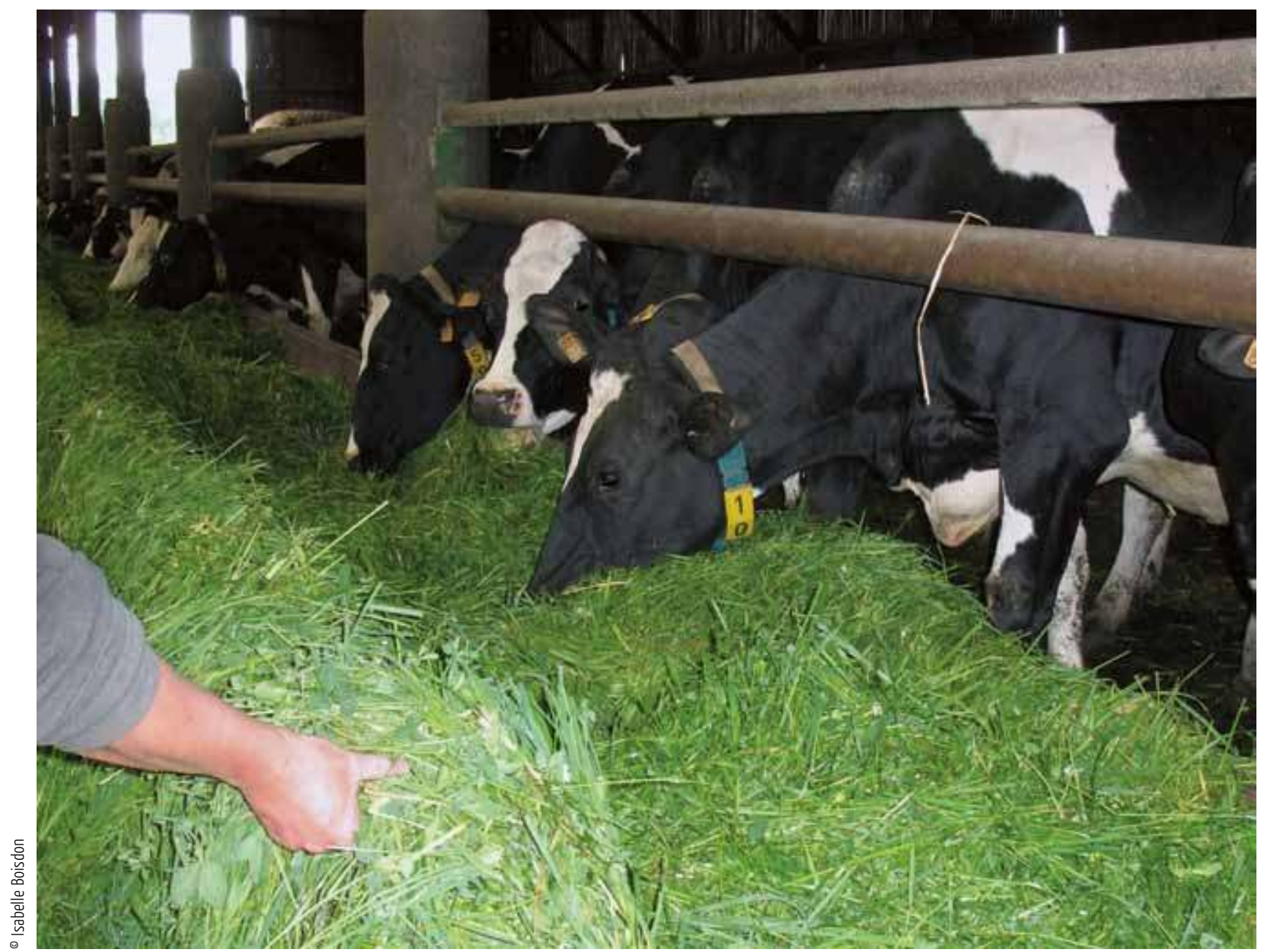

Dans les exploitations en zéro pâturage les animaux productifs restent dans les bâtiments et les hommes récoltent les fourrages nécessaires à leur alimentation. La récolte mécanique des fourrages et leur distribution en bâtiment remplace ainsi le pâturage. 